\title{
miR-25 expression is upregulated in pancreatic ductal adenocarcinoma and promotes cell proliferation by targeting ABI2
}

\author{
HUIMIN LU, LING ZHANG, SHAN LU, DUJIANG YANG, JUN YE, MAO LI and WEIMING HU \\ Department of Pancreatic Surgery, West China Hospital, Sichuan University, Chengdu, Sichuan 610041, PR. China
}

Received September 6, 2018; Accepted November 6, 2019

DOI: $10.3892 / \mathrm{etm} .2020 .8595$

\begin{abstract}
MicroRNAs (miRNAs) are reported to play a critical role in the regulation of cancer cell proliferation; however, the role of microRNA-25 (miR-25) in pancreatic ductal adenocarcinoma (PDAC) remains unclear. In the present study, the role of miR-25 in PDAC cell proliferation was investigated. Upregulated expression of miR-25 was found in PDAC tissues and cell lines by reverse transcription-quantitative PCR. Cell proliferation was significantly enhanced by overexpression of miR-25 as shown by CCK-8 assay results. Meanwhile, overexpression of miR-25 also promoted G1-to-S phase transition of the cell cycle in Aspc-1 cells via flow cytometry analysis. However downregulation of miR-25 inhibited the tumor cell proliferation and cell cycle transition. Online software was used to predict the target gene for miR-25 and luciferase reporter assay confirmed that $\mathrm{Abl}$ interactor $2(A B I 2)$ was a target of miR-25 via direct binding of its 3' untranslated region with miR-25. Moreover, results of the western blot analysis demonstrated that miR-25 negatively regulated the expression of $\mathrm{ABI} 2$ at the protein level. In addition, introduction of ABI2 mRNA into cells overexpressing miR-25 attenuated the carcinogenic effects of miR-25. In conclusion, these findings demonstrate that miR-25 plays an oncogenic role and promotive role in PDAC cell proliferation via targeting of ABI2.
\end{abstract}

\section{Introduction}

Pancreatic cancer is one of the most malignant cancers and is the fourth most common cause for cancer-related deaths worldwide (1). The incidence of pancreatic cancer is rising in many countries. Among the different types of pancreatic cancer, pancreatic ductal adenocarcinoma (PDAC) accounts for more than $80 \%$ of all diagnosed cases of pancreatic cancer; PDAC is considered incurable $(2,3)$. Over the past few

Correspondence to: Dr Weiming Hu, Department of Pancreatic Surgery, West China Hospital, Sichuan University, 37 Hao Guoxue Road, Chengdu, Sichuan 610041, PR. China

E-mail: mfqrp27@163.com

Key words: miR-25, ABI2, pancreatic ductal adenocarcinoma, proliferation, cancer cells decades, significant progress has been made in the diagnosis and treatment of different types of solid cancer, which has greatly increased the chances of patients being cured. However, although the survival rates have increased over the past decade, the prognosis of pancreatic cancer is still poor, with a 5-year-survival rate below $10 \%$ following diagnosis (4). Lack of obvious and unique symptoms and reliable biomarkers for early diagnosis as well as invasive metastasis which results in the reduced sensitivity and response to treatment are the main reason for the poor prognosis. Thus, it is critical to discover novel therapeutic targets and develop novel and effective therapeutic strategies to advance the current therapy for PDAC.

MicroRNAs (miRNAs) are single-stranded small noncoding RNAs which contain approximately 22 nucleotides. miRNAs are reported to play an important role in the modulation of expression levels of their target genes via binding to the 3' untranslated region (5). It has been proven that miRNAs exert a pivotal role in cancer through regulating cancer cell proliferation, differentiation, invasion, migration and cell apoptosis $(6,7)$. In pancreatic cancer cells, various miRNAs were found to act as oncogenic mediators by downregulation or inhibition of the expression of tumor-suppressor genes, while some of the miRNAs serve as tumor suppressors that downregulate expression of oncogenic genes, and thus regulate the proliferation, growth and metastasis of cancer cells. Previous research by other groups showed that the expression levels of miR-20a, miR-21, miR-99a and miR-191 were significantly increased in pancreatic patient serum while miR-375, miR-148, miR-96 and miR-497 expression levels were lower in pancreatic patient serum when compared with levels in normal pancreatic tissues $(8,9)$. Among these miRNAs, miR-25 located on chromosome 7 (7q22), constituted with the miR-106b, miR-93 and miR106b/25 cluster, was reported to play critical roles in different types of human cancers. miR-25 was found to play an oncogenic role in breast cancer (10), ovarian cancer $(11,12)$, gastric cancer $(13,14)$, hepatocellular carcinoma and lung cancer $(15,16)$, where their expression was significantly increased and promoted tumor cell growth and proliferation, while in prostate and colon cancers, miR-25 expression was downregulated and acted as a tumor suppressor (17). However, its role in pancreatic cancer has not been widely investigated.

In the present study, the expression and the biological roles of miR-25 in PDAC tumors and pancreatic cancer cells were 
explored. To date, only one study reported that the expression of miR-25 is increased in pancreatic cancer patient serum and miR-25 was suggested to be used as a potential biomarker for the diagnosis of pancreatic cancer (18). However, the function and the mechanism involved in its regulation of pancreatic cancer proliferation and development are still unknown. The present study revealed that miR-25 expression is much higher in both PDAC tumor tissues and pancreatic cancer cell lines. miR-25 was found to play a promotive role in pancreatic cell proliferation and increased G1-to-S phase transition by targeting $\mathrm{Abl}$ interactor $2(A B I 2)$. Thus, these finding suggest that miR-25 may be a diagnostic biomarker and therapeutic target for PDAC.

\section{Materials and methods}

Tissue samples and cell lines. A total of 25 pairs of pancreatic ductal adenocarcinoma (PDAC) and normal adjacent tissues were collected from patients (12 males and 13 females; age rage 39-76 years) at the West China Hospital (Chengdu, Sichuan, China) between January 2012 and September 2017. Specimens were collected from patients who did not undergo preoperative treatments such as chemotherapy. The present study was approved by the Ethics Committee of the West China Hospital and is in line with the 2004 Helsinki Declaration's code of Ethics. Written informed consents were obtained from all patients or from their legal guardians. Pathological examinations were performed prior to the start of the study to verify the presence of sufficient cancer cells in the tumor tissue samples and that non-cancerous tissues were not contaminated with cancer cells. After surgical resection, tissues were quickly frozen in liquid nitrogen and stored at $-80^{\circ} \mathrm{C}$ until use. Three human PDAC cell lines were kindly provided by the West China Hospital, which included Panc-1, Bxpc-3 and Aspc-1 cells. Sw1990 and HPDE6c7 (human pancreatic non-tumor cell line) cells were obtained from American Type Culture Collection (ATCC). HPDE6c7 was used as a normal control cell line. All human PDAC cell lines were maintained in Dulbecco's modified Eagle's medium (DMEM; Gibco; Thermo Fisher Scientific, Inc.), while the control cell line was cultured in RPMI-1640 medium (Gibco; Thermo Fisher Scientific, Inc.) at $37^{\circ} \mathrm{C}$ in a humidified $5 \% \mathrm{CO}_{2}$ incubator.

Reverse transcription-quantitative PCR (RT-qPCR). Total RNAs were isolated from the PDAC tissues, and HPDE6c7, Panc-1, Bxpc-3, Sw1990 and Aspc-1 cell lines, using TRIzol ${ }^{\circledR}$ reagent (Invitrogen; Thermo Fisher Scientific, Inc.) following the manufacturer's instructions. Then $2 \mu \mathrm{g}$ of total RNA was reverse transcribed into cDNA using the miScript II RT kit (Qiagen). The following thermocycling conditions were used for cDNA synthesis: $37^{\circ} \mathrm{C}$ for $60 \mathrm{~min}$ and $85^{\circ} \mathrm{C}$ for $5 \mathrm{~min}$. qPCR for miR-25 was carried out using a miScript SYBR Green PCR kit (Qiagen). The following thermocycling conditions were used for the qPCR: Initial denaturation at $93^{\circ} \mathrm{C}$ for $15 \mathrm{sec} ; 45$ cycles of $67^{\circ} \mathrm{C}$ for $45 \mathrm{sec}, 93^{\circ} \mathrm{C}$ for $15 \mathrm{sec}, 67^{\circ} \mathrm{C}$ for $1 \mathrm{~min}, 95^{\circ} \mathrm{C}$ for $15 \mathrm{sec}$; a final extension at $75^{\circ} \mathrm{C}$ for $10 \mathrm{~min}$. U6 was used as the internal reference to normalize the miR-25 expression using the $2^{-\Delta \Delta \mathrm{Cq}}$ method (19). The relative expression of AB12 was analyzed by RT-qPCR using SYBR Green PCR Mix (Aidlab) with GAPDH as internal control using the $2^{-\Delta \Delta C q}$ method (19). The sequences for primers used for RT-qPCR were: miR-25-forward, 5'-TCTGGTCTCCCTCACAGGAC-3' and miR-25-reverse, 5'-CATGGGTCGCCTACTCAC-3'; ABI2-forward, 5'-CTCGCAATGTCAAGATCCATTGGC-3' and ABI2-reverse, 5'-TTACTCGCCGCACTGAAGTCAC-3'.

Cell transfection. miR-25 mimics, miR-25 inhibitor, NC and inhibitor NC were commercially obtained from GenePharma (Shanghai, China). miR-25 related solutions $(20 \mu \mathrm{M})$ were prepared by diluting the original stocks with DEPC water. Approximately 5-6x10 cells in logarithmic growth phase were plated into a 6-well plate in complete medium. Aspc-1 cells were transfected with miR-25 mimics $(50 \mathrm{nM}), \mathrm{NC}(50 \mathrm{nM})$, miR-25 inhibitor (150 nM) and inhibitor NC (150 nM) using transfection reagent HiPerFect (Qiagen) with serum-free RPMI-1640 medium.

Cell proliferation assay and cell cycle analysis. Cell proliferation was detected using the CCK-8 assay. Briefly, transfected cells were plated into 96 -well plates with $2 \times 10^{3}$ cells in each well and then the cells were cultured for $0,24,48$ and $72 \mathrm{~h}$. Then, the CCK- 8 assay was performed by adding $10 \mu \mathrm{l}$ of Cell Counting Kit-8 (CCK-8, Beyotime) reagent into each well. The cells in the 96 -well plate were incubated in a $37^{\circ} \mathrm{C}$, $5 \% \mathrm{CO}_{2}$ incubator for $2 \mathrm{~h}$. Then the cell viability/proliferation was determined by measuring of the absorbance at $450 \mathrm{~nm}$ using a microplate reader. 5-Ethynyl-2'-deoxyuridine (EdU) incorporation assay was conducted to assess the proliferation of the transfected cells. Briefly, actively proliferating Aspc-1 cells were incorporated with EdU and then the incorporation was evaluated using a Cell-Light ${ }^{\mathrm{TM}}$ EdU Cell Proliferation Detection kit (RiboBio) according to the manufacturer's protocols. Cell immunostaining was observed and acquired with an epifluorescence microscope (magnification x100; Axioplan II; Carl Zeiss AG) equipped with a charge-coupled device camera. Digital images were analyzed and quantified with Image J 3.1 [National Institutes of Health (NIH), Bethesda, MD, USA). Flow cytometry was performed to analyze the cell cycle. Briefly, transfected cells were digested with trypsin, and then collected by centrifugation at $450 \mathrm{x}$ g for $5 \mathrm{~min}$ at room temperature and washed twice with cold PBS. Cells were resuspended with fixing buffer containing $70 \%$ ethanol and fixed for $30 \mathrm{~min}$ at room temperature. Then cells were stained with $10 \mu \mathrm{g} / \mathrm{ml}$ propidium iodide (PI) (Sigma) for $15 \mathrm{~min}$ at room temperature, and cell cycle stages were analyzed using flow cytometry (BD Biosciences).

Bioinformatics analysis of miR-25 targets. miRanda (http://www.microrna.org/microrna/home.do), miRDB (http://mirdb.org/), miRWalk (http://mirwalk.umm.uni-heidelberg.de/) and TargetScan (http://www.targetscan.org/vert_72/) were used to predict the potential targets of miR-25. Among all the candidates searched, $A B I 2$ (ab1 interactor 2) was predicted to be a target of miR-25, and a highly conserved binding site for miR-25 seed sequence was found in the 3'-UTR region of ABI2 mRNA from position 7039 to 7045 .

Luciferase reporter assay. Luciferase-related ABI2 plasmids were constructed. First, PCR was used to amplify the 3'-UTR sequence of wild-type ABI2 and a target-site mutant, 

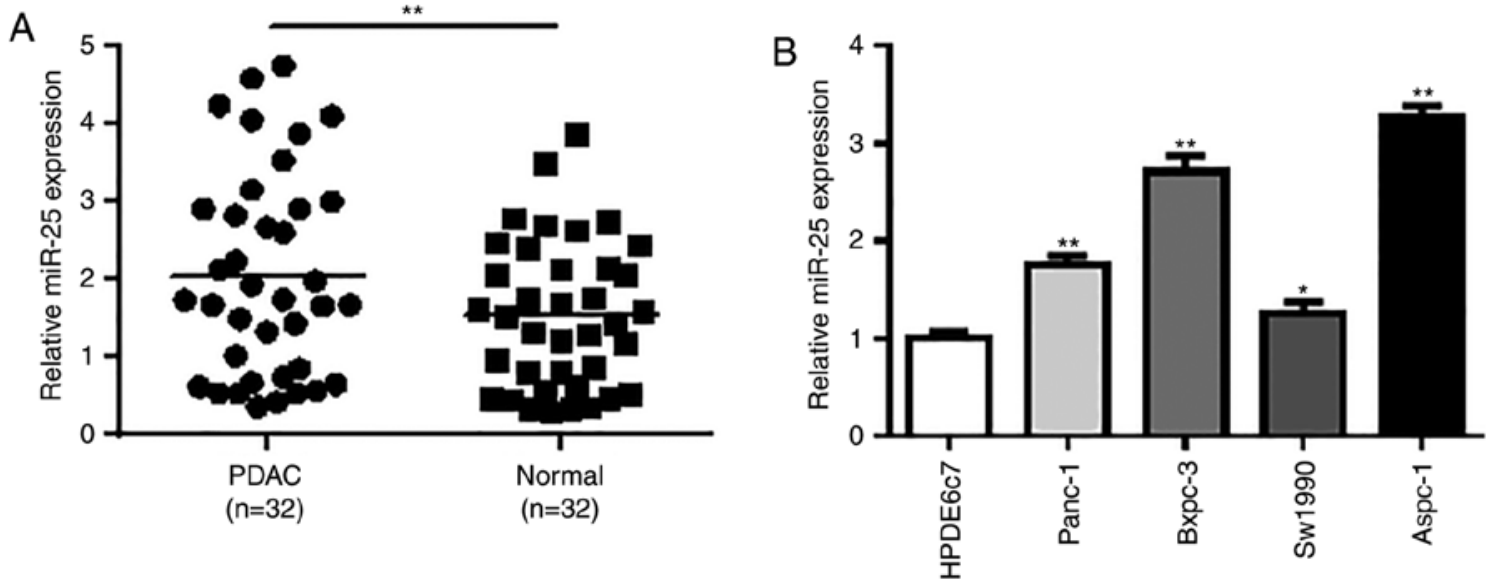

Figure 1. miR-25 is significantly upregulated in PDAC tissues and cell lines. (A) RT-qPCR was performed to detect the relative expression of miR-25 in PDAC and adjacent normal tissue samples. (B) RT-qPCR assay was conducted to determine the relative expression of miR-25 in PDAC cell lines and human pancreatic non-tumor cell line HPDE6c7. Values represent the mean \pm SD. All the experiments were performed in triplicates. ${ }^{*} \mathrm{P}<0.05,{ }^{* * *} \mathrm{P}<0.01 \mathrm{compared}$ with normal tissue or HPDE6c7 cells. PDAC, pancreatic ductal adenocarcinoma; miR, microRNA.
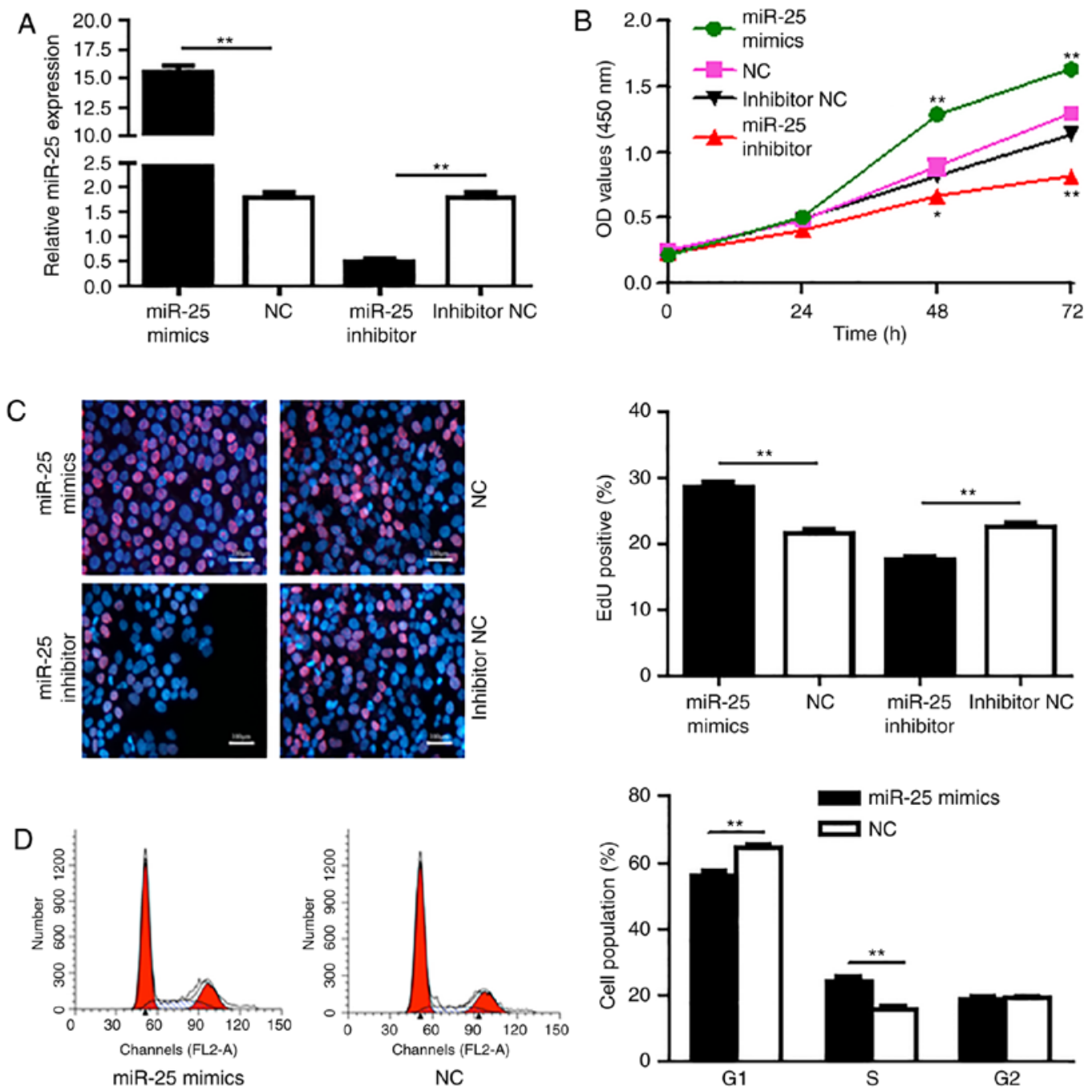

Figure 2. miR-25 promotes the proliferation of PDAC cells. Cell were transfected with miR-25 mimics or inhibitor to alter the expression of miR-25, and then CCK-8 assay was conducted to examine the effect of miR-25 on Aspc-1 cell proliferation. (A) Relative miR-25 expression levels were determined by RT-qPCR after the Aspc-1 cell line was transfected with the indicated plasmids. (B) Aspc-1 cell proliferation after transfection was examined by CCK-8 assay. (C) Aspc-1 cell proliferation was detected by EdU incorporation assay and the staining was observed by immunofluorescence microscopy (magnification, x100). Red indicated the EdU-positive cell, Blue was stained for DAPI. The ratio of EdU-positive cells per optical field (at least 50 fields were acquired) was calculated. (D) Flow cytometric assay showed that the percentage of S phase cells was increased following overexpression of miR-25 and the percentage of G1 phase cells was decreased. Values represent the mean \pm SD. The experiments were repeated for three times and conducted in triplicates. ${ }^{*} \mathrm{P}<0.05,{ }^{* *} \mathrm{P}<0.01$ compared with the control. PDAC, pancreatic ductal adenocarcinoma; miR, microRNA; EdU, 5-ethynyl-2'-deoxyuridine. 
A

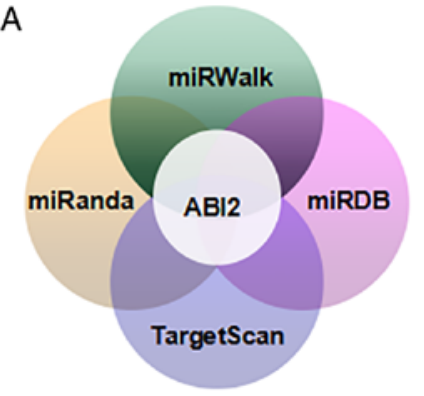

C

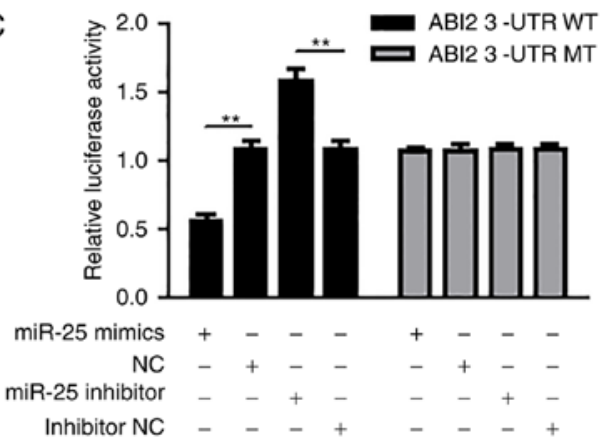

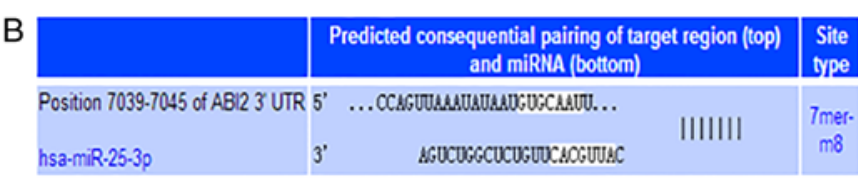
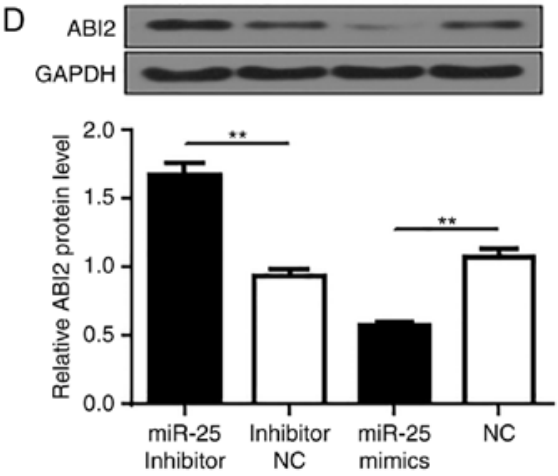

Figure 3. ABI2 is a direct target of miR-25. (A) miRanda (http://www.microrna.org/microrna/home.do), miRDB (http://mirdb.org/), miRWalk (http://mirwalk. umm.uni-heidelberg.de/) and TargetScan (http://www.targetscan.org/vert_72/) were used to search for the potential target genes for miR-25. (B) Potential miR-25 targeting sites and mutant sequences in the ABI2 3'-UTR. (C) Co-transfection of Aspc-1 cells with miR-25 and WT or MT ABI2 3'-UTR luciferase reporter constructs. (D) Aspc-1 cells were co-transfected with miR-25 mimics and miR-25 inhibitors, respectively, and corresponding controls. Western blot assay was performed to examine the protein levels of ABI2. Values represent mean \pm SD. The experiments were repeated three times and conducted in triplicates. ${ }^{* *} \mathrm{P}<0.01$ compared to the control. NC, negative control; miR, microRNA; WT, wild type; MT, mutant; ABI2, Abl interactor 2.

and then the PCR products were ligated into a dual-luciferase reporter vector (Promega), and the products were named as pGL3-ABI2-3'-UTR-WT (wild-type vector) and pGL3-ABI2-3'-UTR-Mut (mutant vector). For cell transfection, Aspc-1 cells at the logarithmic growth phase were seeded into 96 -well plates at a density of $1.5 \times 10^{3}$ cells/well. After being cultured overnight, the Aspc-1 cells were co-transfected with the WT or Mut vector, miR-25 mimics, NC, miR-25 inhibitor, or inhibitor NC using Attractene Transfection Reagent (Qiagen). After transfection for $48 \mathrm{~h}$, the luciferase activity was determined by determining the ratio of firefly to Renilla luciferase activity with a dual-luciferase reporter system (Promega).

Western blot analysis. Cell lysates were prepared by digestion of the collected cells with ice-cold RIPA buffer (Beyotime Institute of Biotechnology) containing10 nM PMSF. The protein concentration of each sample was measured and equal amount of proteins from each sample were separated on $10 \%$ SDS polyacrylamide gels (SDS-PAGE) and then the proteins were transferred to polyvinylidene fluoride membranes. The membranes were blocked with 5\% non-fat milk/TBST for $1 \mathrm{~h}$ and incubated with anti-ABI2 antibody (1:500; cat. no. ab108340; Abcam) at $4^{\circ} \mathrm{C}$ overnight. After being washed with cold TBST 4 times ( 5 min each time), the membranes were incubated with HRP-conjugated goat anti-rabbit immunoglobulin G secondary antibody (1:2,000; cat. no. ab6721; Abcam) for $1 \mathrm{~h}$. The protein expression was visualized via chemiluminescence (Millipore). The ABI2 protein expression level was analyzed using Image J software (NIH). GAPDH (1:1,000; cat. no. ab8245; Abcam) expression served as the control.
Statistical analysis. SPSS 17.0 software (SPSS, Inc.) was used to conduct the statistical analysis and all the data are presented as mean $\pm \mathrm{SD}$. The statistical analysis between two groups was conducted by the independent Student t-test. Differences among more than two groups were analyzed by one-way ANOVA test, followed by Bonferroni's post hoc test. $\mathrm{P}<0.05$ was considered to indicate a statistical significant difference.

\section{Results}

miR-25 is significantly upregulated in PDAC tissues and cell lines. We first analyzed the expression of miR-25 in 25 pairs of PDAC tissues and adjacent normal pancreatic tissues using RT-qPCR. The results showed that miR-25 expression was significantly upregulated in human PDAC tissues when compared with that noted in the adjacent normal tissues $(\mathrm{P}<0.01$; Fig. 1A). Then, we detect the miR-25 expression in different PDAC cancer cell lines and normal cell lines. The RT-qPCR results demonstrated that the miR-25 expression was profoundly elevated in all four PDAC cell lines (Panc-1, Bxpc-3, Aspc-1 and Sw1990) compared with normal HPDE6c7 cells $(\mathrm{P}<0.05, \mathrm{P}<0.01$; Fig. 1B). Among all the four tumor cell lines, Aspc-1 cells had the highest expression of miR-25 and thus Aspc-1 cells were selected for further functional studies.

miR-25 promotes PDAC cell proliferation. Since elevated expression of miR-25 was shown in both PDAC patient tissues and PDAC cancer cell lines, we speculated that miR-25 plays an important role in the regulation of the PDAC cell activities. Hence, the effect of miR-25 on PDAC cell proliferation and growth was detected. To verify the role of miR-25 in the growth 

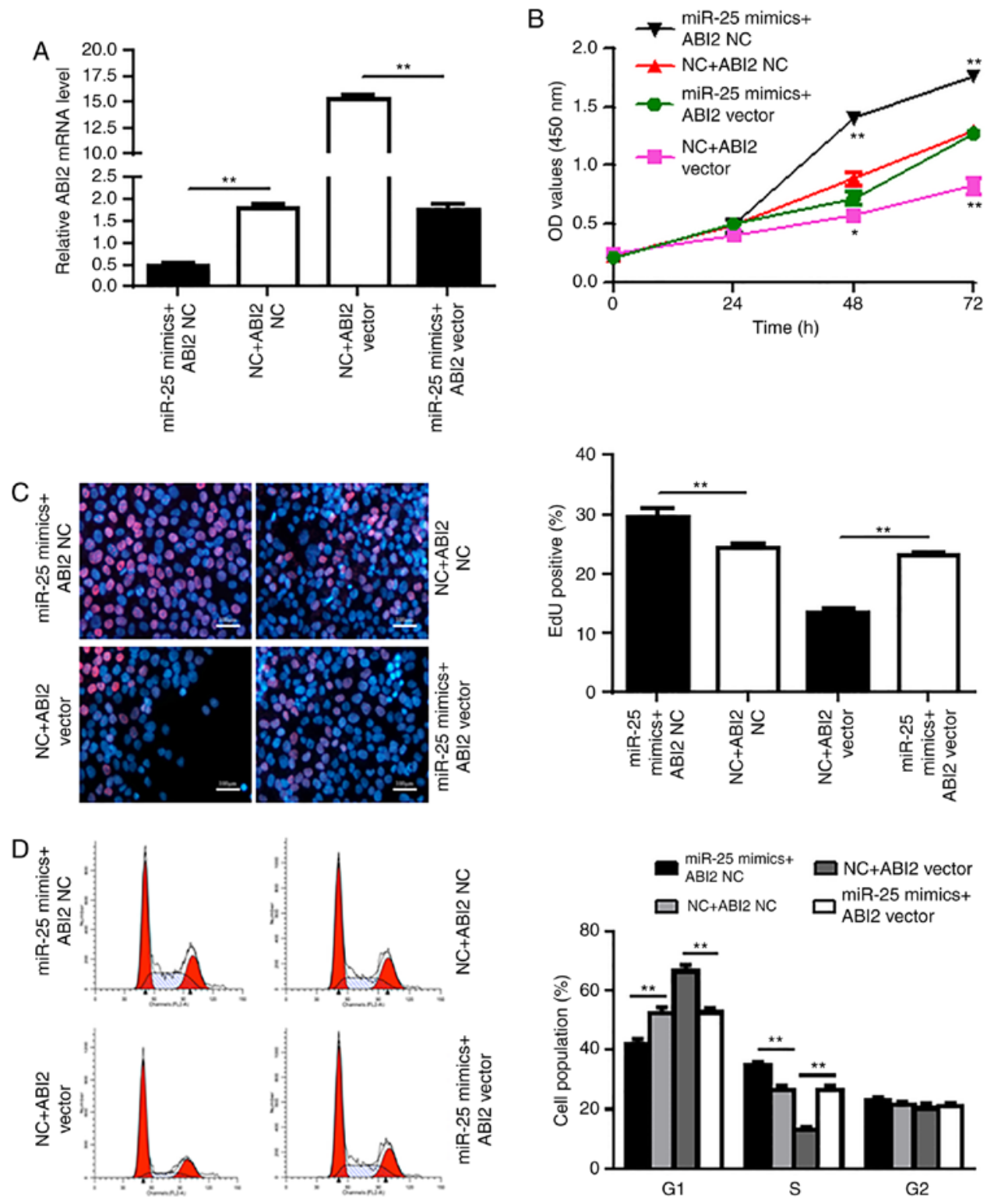

Figure 4. ABI2 overexpression attenuates the promotive effect of miR-25 on PDAC cancer cells. (A) Aspc-1 cells were co-transfected with ABI2 vector and miR-25 mimics, and then RT-qPCR was carried out to detect the relative mRNA expression of ABI2. (B) CCK-8 assay was conducted to determine the proliferation of pretreated Aspc-1 cells. (C) EdU incorporation assay was performed and was monitored by microscopy (magnification, x100). Red indicates EdU-positive cell, Blue was stained for DAPI. The percentage of EdU-positive cells per optical field (at least 50 fields) was counted. (D) Determination of Aspc-1 cell cycle with flow cytometry. Values represent the mean \pm SD. The experiments were repeated three times and conducted in triplicates. ${ }^{*} \mathrm{P}<0.05$, ${ }^{* *} \mathrm{P}<0.01$ compared with control. miR, microRNA; ABI2, Abl interactor 2; EdU, 5-ethynyl-2'-deoxyuridine; NC, negative control.

of PDAC cells, acquisition and loss of function experiments for miR-25 was performed by transfection of Aspc-1 cells with miR-25 mimic, negative control (NC), miR-25 inhibitor and inhibitor NC. RT-qPCR results showed that miR-25 mimic transfection significantly increased the miR-25 level compared with the Aspc-1 cells transfected with $\mathrm{NC}(\mathrm{P}<0.01$; Fig. 2A). While, miR-25 inhibitor transfection markedly decreased the expression of miR-25 compared with cells transfected with inhibitor NC $(\mathrm{P}<0.01)$.

After transfection for 24,48 and $72 \mathrm{~h}$, the CCK- 8 assay was conducted to evaluate the effect of miR-25 on the proliferation of Aspc-1 cells. CCK-8 results showed that the proliferation of Aspc-1 cells in the miR-25 mimetic group was significantly higher at 48 and $72 \mathrm{~h}$ than that noted in the $\mathrm{NC}$ group $(\mathrm{P}<0.01$; Fig. 2B). In addition, the proliferation of Aspc-1 cells in the
miR-25 inhibitor group was markedly lower than that in the inhibitor $\mathrm{NC}$ group $(\mathrm{P}<0.05, \mathrm{P}<0.01)$.

The main event in the $\mathrm{S}$-phase of the cell cycle is DNA replication (synthesis), the aim of which is to produce two identical semi-conserved chromosomes, that transform into another cell cycle and prepare for cell proliferation. Next, EdU incorporation assay was performed to detect the effect of miR-25 on Aspc-1 cell DNA synthesis. The results showed that upregulation of miR-25 resulted in an increase in the population of EdU-positive cells compared to cells treated with the NC-mimics $(\mathrm{P}<0.01)$, while downregulation of miR-25 significantly reduced the population of EdU-positive-cells compared to the cells treated with the inhibitor NC $(\mathrm{P}<0.01)$, indicating that miR-25 promotes the DNA synthesis in Aspc-1 cells (Fig. 2C). 
In addition, malignant cells typically exhibit a dysregulated cell cycle, a key tumor characteristic that promotes tumor proliferation, growth and invasion. Here, we also detected the effect of miR-25 on cell cycle phases in Aspc-1 cells by flow cytometry. As shown in Fig. 2D, Aspc-1 cells were transfected with miR-25 mimic and upregulation of miR-25 was achieved by transfection of miR-25 mimic in Aspc-1 cells and the elevated miR-25 resulted in an increase in the $S$ phase cell population $(\mathrm{P}<0.01)$ and a decrease in the $\mathrm{G} 1$ phase cell population $(\mathrm{P}<0.01)$ compared to the $\mathrm{NC}$ mimic transfected cells These findings confirmed that miR-25 promotes PDAC cell proliferation.

ABI2 is a direct target of $m i R-25$. miRanda (http://www. microrna.org/microrna/home.do), miRDB (http://mirdb.org/), miRWalk (http://mirwalk.umm.uni-heidelberg.de/) and TargetScan (http://www.targetscan.org/vert_72/) were used to search for the potential target genes for miR-25. ABI2 (ab1 interactor2) was predicted to be a target of miR-25, and a highly conserved binding site for miR-25 seed sequence was found in the 3'-UTR region of $A B I 2$ mRNA from position 7039 to 7045 containing the seed sequence (Fig. $3 \mathrm{~A}$ and B). To confirm this prediction, wild-type (WT) or mutated (MT) ABI2 3'-UTR was amplified by PCR and ligated into a luciferase reporter vector and assayed for detection of luciferase activity. As shown in Fig. 3C, overexpression of miR-25 profoundly inhibited the 3'-UTR of WT rather than MT, whereas inhibition of miR-25 significantly promoted the 3'-UTR of WT rather than MT $(\mathrm{P}<0.01)$. In addition, consistent with the dual luciferase assay results, immunoblotting results showed that upregulation of miR-25 reduced ABI2 protein levels, while inhibition of miR-25 significantly increased $\mathrm{ABI} 2$ protein levels $(\mathrm{P}<0.01)$. (Fig. 3D). Thus, these observations indicate that miR-25 negatively regulates the expression of $\mathrm{ABI} 2$ by directly binding to its 3'-UTR.

ABI2 overexpression attenuates the oncogenic effect of $m i R-25$. To further investigate the functional relationship between miR-25 and ABI2, we designed the ABI2 vector and transfected Aspc-1 cells with this vector and miR-25 mimic, which was confirmed by RT-qPCR (Fig. 4A). The ABI2 vector was found to significantly attenuate the carcinogenic effect of miR-25 in Aspc-1 cells $(\mathrm{P}<0.05, \mathrm{P}<0.01)$ (Fig. 4B-D). These data indicate that miR-25 promotes Aspc-1 cell proliferation by negatively regulating its target gene $A B I 2$.

\section{Discussion}

miRNAs are small non-coding RNAs, that have been explored and proven to serve as potential diagnostic biomarker and prognostic factor in multiple types of cancers (20). In addition, miRNAs may play an oncogenic role or tumor-suppressor role in different tumors to regulate cancer cell proliferation, growth and tumor metastasis including pancreatic cancer $(7,20)$. In pancreatic cancer, several serum miRNAs have been investigated and suggested as biomarker for both diagnosis and prognosis and the expression profile of these miRNAs has been reported to be associated with the development and progression of pancreatic cancer $(21,22)$. Among these miRNAs, miR-29a and miR-21 have been reported to promote pancreatic cancer growth (23), while miR-429 plays an inhibitory role in pancreatic ductal adenocarcinoma growth via targeting of TBK1 (23). Due to the high mortality rate of PDAC, a highly malignant disease, discovery of novel biomarkers of PDAC and investigation of new therapeutic targets for PDAC are critical and important for improving the survival time and treatment efficacy.

Among all the miRNAs, miR-25 is one of the most researched and well-described miRNA related to different human cancers. It is 22 nucleotides in length and has been reported to be overexpressed in different type of human carcinomas which include breast cancer (10), ovarian cancer $(11,12)$, gastric cancer $(13,14)$, hepatocellular carcinoma and lung cancer $(15,16)$. However, the expression of miR-25 in PDAC tumor tissue and its biological function as well as the molecular mechanism involved in its biological role are still under investigation. In the present study, our results showed that miR-25 serves as an important regulator in the progression of PDAC. First, our data demonstrated that the expression of miR-25 was significantly higher in PDAC tissues when compared with normal tissues. Moreover, expression of miR-25 was also upregulated in PDAC cell lines compared with that noted in the normal immortalized human pancreatic duct epithelial cell line HPDE6c7 which has been widely used for comparison with PDAC cells in many research studies. Furthermore, upregulation of miR-25 significantly enhanced cell proliferation and G1-to-S phase transition in Aspc-1 cells, while downregulation of miR-25 inhibited the tumor cell proliferation and cell cycle transition. These data revealed for the first time that miR-25 plays an oncogenic role and promotes pancreatic cell proliferation and growth through regulation of the pancreatic cancer cell cycle, indicating its key regulatory role in promotion of the progression of PDAC.

To further investigate the miR-25 target in PDAC, luciferase reporter assay was performed. The results revealed that miR-25 directly binds to the 3 ' untranslated region of ABI2. In addition western blot analysis showed that miR-25 downregulated $\mathrm{ABI} 2$ expression at the protein level. ABI-2, a member of the ABI family of adaptor proteins, has been proven to be involved in signaling pathways involving tyrosine kinase and Rac GTPase. One previous study showed that deficiency of $\mathrm{ABI} 2$ led to defects in cell migration (24). In the present study, introduction of ABI 2 mRNA into cells overexpressing miR-25 attenuated the miR-25 overexpression-induced cell proliferation and cell cycle regulation. In conclusion, these results demonstrate that miR-25 is an oncogenic miRNA that promotes PDAC cell proliferation by targeting ABI2. Altogether, our study indicated that miR-25 may have the potential to be investigated as a biomarker for the diagnosis of PDAC and its oncogenic role in PDAC suggest that it may be explored as a potential therapeutic target for the treatment of PDAC. However, there are still limitations in the present research study. Only one PDAC cell line was used in the functional studies, and more cell lines will be needed to confirm the role of miR-25 in the regulation of PDAC cell function and activity. Moreover, an in vivo model should be established in the future to further verify the function of miR-25 in PDAC.

\section{Acknowledgements}

Not applicable 


\section{Funding}

No funding was received.

\section{Availability of data and materials}

The datasets used and/or analyzed during the current study are available from the corresponding author on reasonable request.

\section{Authors' contributions}

WH and HL conceived and designed the study. HL, LZ, SL, DY, JY and ML performed the experiments. WH and HL wrote the manuscript. LZ, SL, DY, JY and ML reviewed and edited the manuscript. All authors read and approved the manuscript and agree to be accountable for all aspects of the research in ensuring that the accuracy or integrity of any part of the work are appropriately investigated and resolved. All authors read and approved the final manuscript.

\section{Ethics approval and consent to participate}

The present study was approved by the Ethics Committee of the West China Hospital. All patients and healthy volunteers provided written informed consent prior to their inclusion within the study.

\section{Patient consent for publication}

Not applicable.

\section{Competing interests}

The authors declare that they have no competing interests.

\section{References}

1. Hariharan D, Saied A and Kocher HM: Analysis of mortality rates for pancreatic cancer across the world. HPB (Oxford) 10 : 58-62, 2008.

2. Garrido-Laguna I and Hidalgo M: Pancreatic cancer: From state-of-the-art treatments to promising novel therapies. Nat Rev Clin Oncol 12: 319-334, 2015.

3. Adamska A, Domenichini A and Falasca M: Pancreatic Ductal Adenocarcinoma: Current and Evolving Therapies. Int J Mol Sci 18: E1338, 2017.

4. Vincent A, Herman J, Schulick R, Hruban RH and Goggins M: Pancreatic cancer. Lancet 378: 607-620, 2011.

5. Garzon R, Marcucci G and Croce CM: Targeting microRNAs in cancer: Rationale, strategies and challenges. Nat Rev Drug Discov 9: 775-789, 2010.

6. Friedman RC, Farh KKH, Burge CB and Bartel DP: Most mammalian mRNAs are conserved targets of microRNAs. Genome Res 19: 92-105, 2009.
7. Garofalo $\mathrm{M}$ and Croce CM: microRNAs: Master Regulators as Potential Therapeutics in Cancer. Annu Rev Pharmacol Toxicol 51: 25-43, 2011.

8. Zhang Y, Li M, Wang H, Fisher WE, Lin PH, Yao Q and Chen C: Profiling of 95 microRNAs in pancreatic cancer cell lines and surgical specimens by real-time PCR analysis. World J Surg 33: 698-709, 2009.

9. Khan MA, Zubair H, Srivastava SK, Singh S and Singh AP: Insights Into the Role of microRNAs in Pancreatic Cancer Pathogenesis: Potential for Diagnosis, Prognosis, and Therapy. Adv Exp Med Biol 889: 71-87, 2015.

10. Wang Z, Wang N, Liu P, Chen Q, Situ H, Xie T, Zhang J, Peng $\mathrm{C}$, Lin $\mathrm{Y}$ and Chen J: MicroRNA-25 regulates chemoresistance-associated autophagy in breast cancer cells, a process modulated by the natural autophagy inducer isoliquiritigenin. Oncotarget 5: 7013-7026, 2014.

11. Zhang H, Zuo Z, Lu X, Wang L, Wang H and Zhu Z: miR-25 regulates apoptosis by targeting Bim in human ovarian cancer. Oncol Rep 27: 594-598, 2012.

12. Feng S, Pan W, Jin Y and Zheng J: miR-25 promotes ovarian cancer proliferation and motility by targeting LATS2. Tumour Biol 35: 12339-12344, 2014.

13. Zhao $\mathrm{H}$, Wang Y, Yang L, Jiang $\mathrm{R}$ and Li W: miR-25 promotes gastric cancer cells growth and motility by targeting RECK. Mol Cell Biochem 385: 207-213, 2014.

14. Li BS, Zuo QF, Zhao YL, Xiao B, Zhuang Y, Mao XH, Wu C, Yang SM, Zeng H, Zou QM, et al: MicroRNA-25 promotes gastric cancer migration, invasion and proliferation by directly targeting transducer of ERBB2, 1 and correlates with poor survival. Oncogene 34: 2556-2565, 2015.

15. Li Q, Zou C, Zou C, Han Z, Xiao H, Wei H, Wang W, Zhang L, Zhang X, Tang Q, et al: MicroRNA-25 functions as a potential tumor suppressor in colon cancer by targeting Smad7. Cancer Lett 335: 168-174, 2013.

16. Wang C, Wang X, Su Z, Fei H, Liu X and Pan Q: MiR-25 promotes hepatocellular carcinoma cell growth, migration and invasion by inhibiting RhoGDI1. Oncotarget 6: 36231-36244, 2015.

17. Caiazza C and Mallardo M: The Roles of miR-25 and its Targeted Genes in Development of Human Cancer. MicroRNA 5: 113-119, 2016.

18. Deng T, Yuan Y, Zhang C, Zhang C, Yao W, Wang C, Liu R and $\mathrm{Ba}$ Y: Identification of Circulating MiR-25 as a Potential Biomarker for Pancreatic Cancer Diagnosis. Cell Physiol Biochem 39: 1716-1722, 2016.

19. Livak KJ and Schmittgen TD: Analysis of relative gene expression data using real-time quantitative PCR and the $2-\Delta \Delta C$ T method. Methods 25: 402-408, 2001.

20. Hayes J, Peruzzi PP and Lawler S: MicroRNAs in cancer: Biomarkers, functions and therapy. Trends Mol Med 20: 460-469, 2014.

21. Song W, Li Q, Wang L and Wang L: Modulation of FoxO1 expression by miR-21 to promote growth of pancreatic ductal adenocarcinoma. Cell Physiol Biochem 35: 184-190, 2015.

22. Sun XJ, Liu BY, Yan S, Jiang TH, Cheng HQ, Jiang HS, Cao Y and Mao AW: MicroRNA-29a Promotes Pancreatic Cancer Growth by Inhibiting Tristetraprolin. Cell Physiol Biochem 37: 707-718, 2015.

23. Song B, Zheng K, Ma H, Liu A, Jing W, Shao C, Li G and Jin G: miR-429 determines poor outcome and inhibits pancreatic ductal adenocarcinoma growth by targeting TBK1. Cell Physiol Biochem 35: 1846-1856, 2015.

24. Grove M, Demyanenko G, Echarri A, Zipfel PA, Quiroz ME, Rodriguiz RM, Playford M, Martensen SA, Robinson MR, Wetsel WC, et al: ABI2-deficient mice exhibit defective cell migration, aberrant dendritic spine morphogenesis, and deficits in learning and memory. Mol Cell Biol 24: 10905-10922, 2004. 